\title{
Study of stellar activity through transit mapping of starspots
}

\author{
Adriana Valio ${ }^{1}$ \\ ${ }^{1}$ Center for Radioastronomy and Astrophysics, Mackenzie University \\ São Paulo, Brazil \\ email: avalio@craam.mackenzie.br
}

\begin{abstract}
During the eclipse of a planet, spots and other features on the surface of the host star may be occulted. This will cause small variations in the light curve of the star. Detailed studies of these variations during planetary transits provide a wealth of information about the starspots properties such as size, position, temperature (i.e. intensity), and magnetic field. If observation of multiple transits is available, the spots lifetime can be estimated. Moreover it may also be possible to determine the stellar rotation and whether differential rotation is present. Here, the study is performed using a method that simulates the passage of a planet (dark disk) in front of a star with multiple spots of different sizes, intensities, and positions on its surface. The data variations in the light curve of the star are fit using this method, yielding the starspots properties. Results are presented for solar-like stars, such as the active star CoRoT-2a.
\end{abstract}

Keywords. Starspots, stellar activity, planetary transit, exoplanet

\section{Introduction}

Presently, there are almost 500 known planets orbiting other stars other than our Sun, discovered during the past 15 to 18 years. About one fifth of them (101 planets, or 20\%) eclipses their host star. During one of these transits, the planet may pass in front of a spot group and cause a detectable signal in the light curve of the star, of the order of a few tenths of a percent.

A method that simulates planetary transits was developed by Silva (2003), where the planet is used as a probe to investigate the presence and characteristics of features on the surface of the star, such as spots. The physical parameters of these spots resulting from this study are size, intensity (or temperature), location on the surface of the star (longitude and latitude), and lifetime, among others. Stellar properties such as rotation (Silva-Valio 2008) and differential rotation can also be estimated.

Lightcurves of individual planetary transits are fit by the model of a star with several round spots on its surface, and the spots properties is obtained by a $\chi^{2}$ minimization technique. The star is modeled as a $2-\mathrm{D}$ matrix with a given limb darkening (linear or quadratic profiles), or a real image of the Sun can be used. On the otherhand, the planet is modeled as an opaque disk of radius $r / R_{s}$, where $R_{s}$ is the stellar radius. Every 2 min (or the desired time interval), the position of the planet in its orbit is calculated. The orbit is assumed circular with radius $a_{\text {orb }} / R_{s}$ and inclination angle $i$. By summing over all the pixels in the image, the lightcurve intensity is obtained at every time interval.

At any given transit, the number of spots on the stellar surface is variable. Each spot is characterized by 3 parameters - Intensity: measured with respect to stellar maximum 

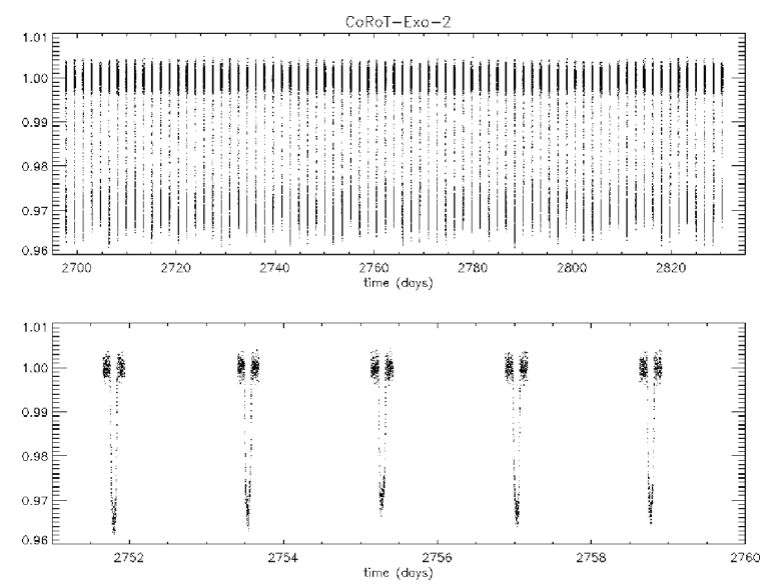

Figure 1. Top: Lightcurve of the 77 transits of CoRoT-2b. Bottom: Examples of 5 of these transits.

intensity (at disk center); Size: measured in units of planetary radius; and Position: Latitude (restricted to the transit band) and Longitude.

Here the technique of spot mapping is applied to the case study of CoRoT-2, the second planet-hosting-star found by the CoRoT satellite (Auvergne et al. 2009). CoRoT-2 is a G7V star of $0.97 M_{\text {Sun }}$ and $0.902 R_{\text {Sun }}$, with an effective temperature of $5625 \mathrm{~K}$. It is a fairly young star, with an estimated age of less than 0.5 Gyr. The planet CoRoT-2b is a hot Jupiter with $M \sin (i)=3.31 M_{J u p}$, radius of $1.465 R_{J u p}$, orbiting the star every 1.743 day at a distance of only $6.7 R_{s}$ (approximately 0.03 A.U.) in and inclined orbit of $87.84^{\circ}$ (Alonso et al. 2008). This data was previously analyzed by Lanza et al. (2009); Wolter et al. (2009) and Huber et al. (2010).

\section{Transit mapping}

Because CoRoT observes the same region of the sky for up to 150 days, a total of 77 transits of CoRoT-2b were observed, which are shown in the top panel of Figure 1. Due to its size, orbital radius, and inclination angle, the planet obscures a band on the stellar surface of $20^{\circ}$ centered at $-14.6^{\circ}$ latitude.
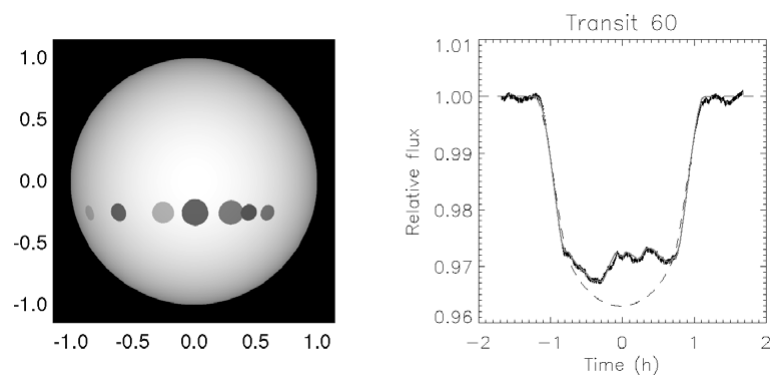

Figure 2. Left: modeled star with 7 spots. Right: Lightcurve of transit 60 , with the bars representing the uncertainty of the CoRoT-2 data, $\sigma=0.0006$. The solid gray line represents the best fit to the data, where 7 spots were considered. The dashed line is the result from a model of a spotless star. 

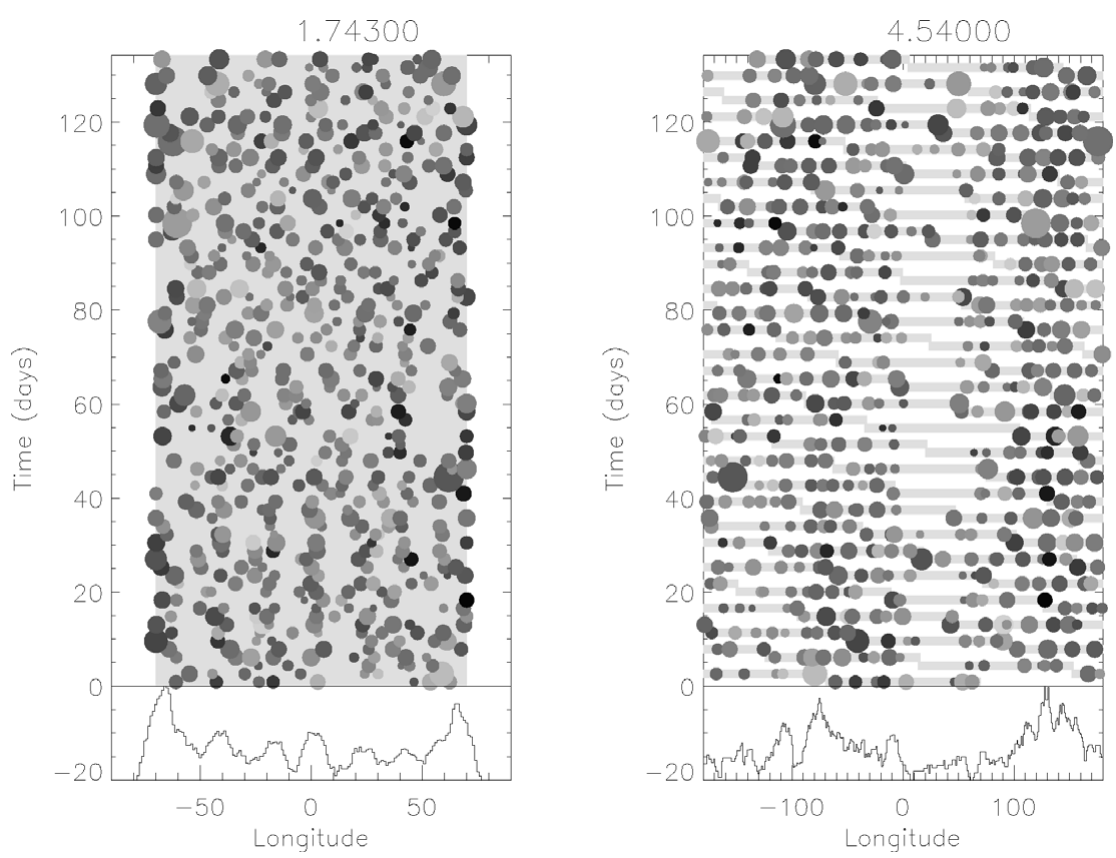

Figure 3. Left: Spot map of the stellar surface considering a reference frame fixed with respect to the observer, that is spots position (topocentric longitudes) at every transit. Right: Same as in left panel but in the rotating frame of the star. The curves on the bottom of each panel represent the time integrated flux deficit of the spots for each longitude.

The presence of spots on the stellar surface causes the transit to be less deep, because the region been eclipsed by the planet has a smaller brightness than otherwise (see right panel of Figure 2). Up to a maximum of 9 spots are considered in each transit, with their longitudes constrained between $\pm 70^{\circ}$ due to the very steep variation of the lightcurve during the times of ingress and egress of the planet. The radius, $R$, and intensity, $I$, of the spots are restricted between 0 and 1 , in units of planet radius $\left(R_{p}\right)$ and central stellar brightness $\left(I_{c}\right)$. An example of the fit for the 60 th transit with 7 spots is shown in Figure 2.

The analysis of the 77 transits has been presented by Silva-Valio et al. (2010a). A total of about 600 spots were modeled, where only spots with a flux deficit, defined as $F=\left(1-I / I_{c}\right) \times\left(R / R_{p}\right)^{2}$, larger than 0.02 in relative units were considered. The fitting of the transits yields spots sizes ranging from 0.2 to $0.7 R_{p}$, with an average value of $0.46 \pm 0.11 R_{p}(\sim 100,000 \mathrm{~km})$, whereas the intensity varied from 0.3 to 0.8 $I_{c}$, with a mean of $0.55 \pm 0.13 I_{c}$. This intensity can be translated to temperature by assuming black-body emission for both the stellar photosphere and the spot. Considering an effective temperature of $5625 \mathrm{~K}$ for the stellar photosphere, the mean spot temperature found was $4700 \pm 300 \mathrm{~K}$.

Silva-Valio et al. (2010a) also found that the total surface area of the star covered by the spots, within the transit band, ranges from 10 to $20 \%$. For the Sun, the spot covered area is less than $1 \%$. However, one must bear in mind that CoRoT-2 is a young and very active star, with an age less than one tenth that of the Sun.

Maps of the stellar surface region occulted by the planet can be reproduced for each transit. Such map is shown in the left panel of Figure 3, where the best result for each transit is shown as filled circles representing spots of a certain relative size and 


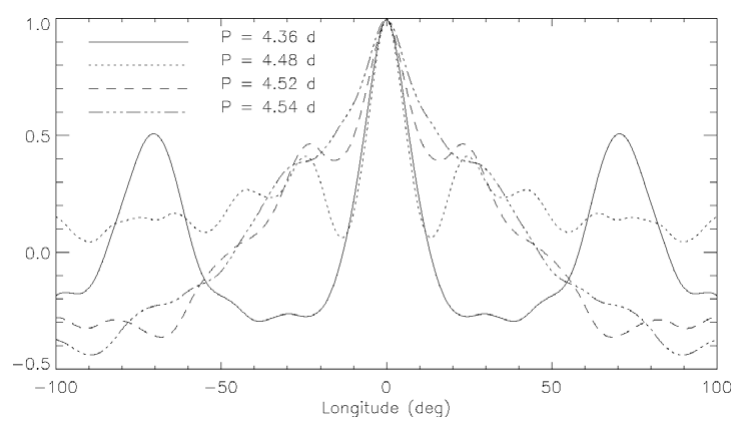

Figure 4. Auto-correlation functions of the flux deficit integrated in time for each longitude bin for stellar rotation periods of $4.36,4.48,4.52$, and 4.54 days.

intensity placed at their fitted longitude for each transit in time (vertical axis). The shaded region in the background represents the visible stellar hemisphere (within $\pm 70^{\circ}$ longitudes) during each transit. The fitted longitudes shown in the left panel of Figure 3 are the topocentric longitude, measured in a fixed reference frame with respect to the observer, where zero longitude coincides with the projection of the line-of-sight at mid transit.

To study the temporal evolution of the spots, it is necessary to associate whether some of the spots are detected again in a posterior transit. For that, it is necessary to use a reference frame that rotates with the star. In this case, the spots will be identified by their common rotation longitude. The rotation longitude, $\beta_{\text {rot }}$, may be obtained from the topocentric longitude, $\beta_{\text {topo }}$, once the stellar rotation period, $P_{\text {star }}$, is known.

$$
\beta_{\text {rot }}=\beta_{\text {topo }}-360^{\circ} \frac{n P_{\text {orb }}}{P_{\text {star }}}
$$

where $P_{\text {orb }}=1.743$ day is the planet orbital period, $P_{\text {star }}$ the stellar rotation period, and $n$ the transit number. As a first estimate for the rotation period within the transit band, the average period of the star of 4.54 days obtained from the out-of-transit data is used (Alonso et al. 2008). A map of the spotted stellar surface in time (for every transit) versus rotational longitudes is shown in the right panel of Figure 3. The figure clearly shows that there is a longitude band within 0 and $40^{\circ}$ where there are almost no spots visible during the total observation period of 134 days.

\section{Differential rotation}

The differential rotation profile of the stellar surface may be calculated from the measured rotation period within a given latitude band and the average rotation of the star. If we are to consider that the rotation period at $-14.6^{\circ}$ is equal to the mean rotation period of the star of $4.54 \mathrm{~d}$, this implies that this star presents no differential rotation. However, this is an active star with many spots on its surface at any given time, therefore a magnetic dynamo process must be taking place within the star. Thus one possibility is that the rotation period within the transit band is different than 4.54 days, and what is seen here is something similar to the counterpart of a coronal hole observed on our Sun.

Coronal holes are regions of open magnetic field lines in the Sun, with lower temperature and density than the rest of the corona. They present smaller differential rotation, sometimes nonexistent, than the photosphere where the spots are located. Also from 


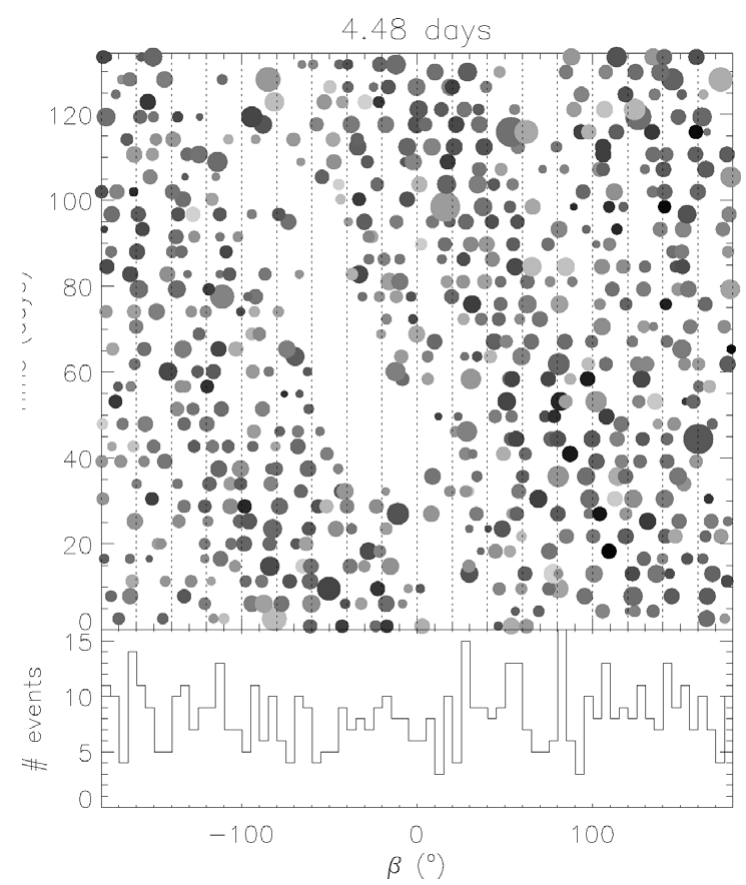

Figure 5. Same as Figure 3 for a stellar rotation period of 4.48 days within the transit latitudes.

solar observations, it is known that the photosphere below the coronal holes has no spot. Therefore, we will assume that the spotless photosphere within $0-40^{\circ}$ longitudes of CoRoT-2 is the surface counterpart of a coronal hole that reaches near the equator of the star.

Thus the problem now is to determine the true rotation period of the transit band. For this, we calculated the stellar surface map for different rotation periods from 2 to 6 days, every 0.01 day. For each map (or rotation period), the flux deficit due to spots for a given longitude is summed over all transits. Next, the auto-correlation of the flux deficit as a function of longitude is calculated, and the curve with the smaller width is assumed to be the best estimate of the stellar rotation period within $-25^{\circ}$ and $-5^{\circ}$ latitudes. Examples of the auto-correlation functions for 4 rotation periods of the transit band are shown in Figure 4.

The auto-correlation function of the time integrated flux deficit was found to be the narrowest for a rotation period of 4.48 days (see Figure 4 ). Thus hereafter, the rotation period of the star within the transit latitudes of $-14.6 \pm 10^{\circ}$ is assumed to be 4.48 days, and the corresponding stellar surface map is shown in Figure 5.

Now, the differential rotation of the star can be estimated, this is assumed to follow the same functional form as that of the Sun, where the rotational angular velocity, $\Omega$, is given by $\Omega=A-B \cos ^{2} \theta$, where $\theta$ is the co-latitude, and in the case of the Sun $A=14.55^{\circ}$ day $^{-1}$ and $B=2.87^{\circ}$ day $^{-1}$. For CoRoT-2 these constants are estimated by considering that the rotation period at co-latitude of $75.4^{\circ}=90^{\circ}-14.6^{\circ}$ is 4.48 days, and that the rotation period averaged over all latitudes is 4.54 days. These two values yield $A=80.5^{\circ}$ day $^{-1}$ and $B=2.4^{\circ}$ day $^{-1}$, therefore: 


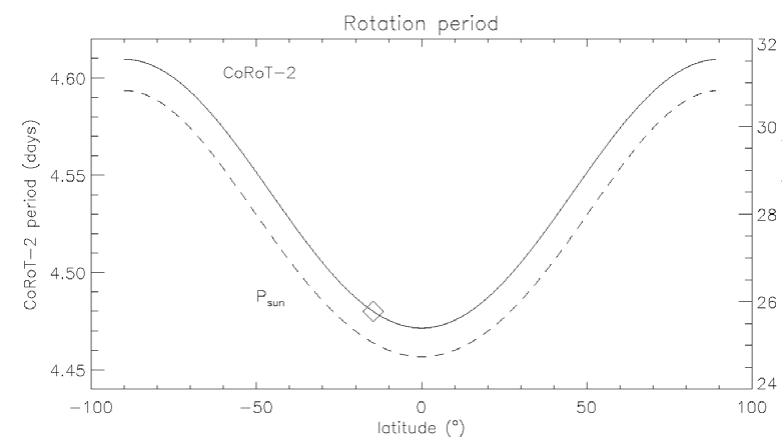

Figure 6. Differential rotation profile, that is, rotation period versus latitude, for CoRoT-2 (solid) and the Sun (dashed, scale on the right of the figure).

$$
P_{\text {CoRoT }-2}=\frac{360^{\circ}}{80.5-2.4 \cos ^{2}\left(90^{\circ}-\alpha\right)} \text { days, }
$$

where $\alpha$ is the latitude. The rotation period as a function of latitude is plotted in Figure 6 . The solar differential rotation is also plotted as a dotted line. The transit latitude value is marked by the diamond, that is, the period of 4.48 days at $\alpha=-14.6^{\circ}$.

\section{Discussion and conclusions}

A total of 77 consecutive transits were observed for the planet hosting star CoRoT-2. This was possible because the orbital period of the planet is only 1.743 day. By modeling small variations in the light curve observed during transits as due to star spots, it was possible to infer the presence of at about 600 spots on the stellar surface during these 134 days of observation.

The modelling of spots yields its physical characteristics such as size, temperature, location, surface area coverage, etc (Silva 2003; Silva-Valio et al. 2010a). If multiple transits are available, also the stellar rotation can be obtained (Silva-Valio 2008), and even stellar differential rotation such as the study presented here.

To estimate the differential rotation of CoRoT-2a, first it was necessary to determine the rotation period within the given latitude band. This was obtained by calculating the time integrated spot flux deficit for each longitude for several values of stellar rotation. Then, the auto-correlation of the flux deficit function with the narrowest peak was chosen as that representing the true rotation period within the $-14.6 \pm 10^{\circ}$ latitudes. This procedure yields a value of 4.48 days for this rotation period.

The differential rotation profile obtained here shows that close to the equator, the star rotates faster, with a period of $4.47 \mathrm{~d}$, while the longest period of 4.61 days is found at the poles. Using the values listed in Eq. (3.1) resulted in $\Delta \Omega=\Omega_{e q}-\Omega_{\text {pole }}=0.042$ $\mathrm{rad} / \mathrm{d}$. This corresponds to $\left.\left(P_{\text {pole }}-P_{e q}\right) /<P\right\rangle=3 \%$, where $\langle P\rangle$ is the mean rotation period. From out-of-transit data analysis, Lanza et al. (2009) estimated a lower limit to the amplitude of the relative differential rotation of only $\sim 0.7-1 \%$ for this same star, while from a model with three spots only, Fröhlich et al. (2009) find a mean differential rotation of $0.11 \mathrm{rad} / \mathrm{d}$ and a maximum differential rotation of $0.129 \mathrm{rad} / \mathrm{d}$. As can be seen, the differential rotation estimated here is a intermediate value between both estimates.

Once the rotation period of the star within the transit latitude band is known, it is possible to identify the same spot on multiple transits and study their temporal evolution. 
However, we refer the reader to the work of Silva-Valio and Lanza (2010b) for a detailed analysis of the temporal behavior of the spots on the photosphere of CoRoT-2.

\section{References}

Alonso, R., Auvergne, A., \& Baglin, A. et al. 2008, Astron. Astrophys, 482, L21

Auvergne, M., et al. 2009, Astron. Astrophys, 506, 411

Fröhlich, H.-E., Küker, M., Hatzes, A. P., \& Strassmeier, K. G. 2009, Astron. Astrophys, 506, 263

Huber, K. F., Czesla, S., Wolter, U., \& Schmitt, J. H. M. M. 2010, Astron. Astrophys, 514, A39

Lanza, A. F., Pagano, I., \& Leto, G., et al. 2009, Astron. Astrophys, 493, 193

Silva, A. R. V. 2003, Astrophys. J., 585, L147

Silva-Valio, A. 2008, Astrophys. J., 683, L179

Silva-Valio, A., Lanza, A. F., Alonso, R., \& Barge, P. 2010, Astron. Astrophys, 510, A25

Silva-Valio, A. \& and Lanza, A. F. 2010, Astron. Astrophys (submitted)

Wolter, U., Schmitt, J. H. M. M., Huber, K. F., Czesla, S., Müller, H. M., Guenther, E. W., \& Hatzes, A. P. 2009, Astron. Astrophys, 504, 561

\section{Discussion}

DUPREE: I would like follow up on your detection of coronal holes and ask, A, what the lifetime was during the whole COROT point of 180 days which seems anomalous or very long compared to the sun? And can you follow up with Chandra observations or Hubble observations to actually detect modulation and the diminution, X-ray flux, and symmetries of lines that might indicate out flow?

VALIO: Yes, it lasts for the whole 140 days. Maybe at the end there might be some evidence that it's kind of not there anymore but yes, yes. And for the second comment, that's a great idea.

KitiashVILI: Very interesting method you show. My question is about hot Jupiters which you observe like a transit across a star. No one [INAUDIBLE] models of hot Jupiters hot Jupiters have very massive atmosphere. How will it effect the atmosphere on your results?

VALIO: Okay. For this one, I'm just modeling as a black - completely black disk, absolutely no emission. And of course you can change that and add a transparency like varying towards the edge of the planet, but I haven't done that. But I don't think that's going to be - unless it's very transparent, it's - I don't think it's going to matter much for the spot study. It will matter if you're analyzing the shape of the transit and things like that.

JARDINE: Do you have any plans to look at the transiting planets that are in highly inclined orbits because that will give you access to different latitudes in the star?

VALIO: Yes, yes, that's next. Hopefully try and measure the angle.

KLIMCHUK: Is it your technique sensitive enough that you could say something about possibly penumbrae?

VALIO: Maybe if they are big as I was hoping, I could use this for COROT-7 or other smaller planets. But the notes were the COROT, again I can't look in the vision transits. 
But for Kepler, I did a very brief simulation. If you get a earth-size planet, then you get all this - like on the solar planet, not on this, there you have all the activity regions and the penumbra; and you do get the structures. So, yes, with this model planet, you can do that. 